\title{
DESAFÍOS AMBIENTALES Y SOCIALES FRENTE AL CAMBIO CLIMÁTICO
}

\author{
María del Mar Delgado', María José Ambrosio Albala², Cecilia Riccioli Giuliarini ${ }^{3}$
}

Fecha de recepción: $\quad 09-08-13$

Fecha de aceptación: 03-02-14

\section{Resumen}

Los modelos actuales de desarrollo y explotación de recursos naturales están incrementando las tensiones y los impactos que sufren estos recursos. El cambio climático está intensificando esta situación. El presente artículo analiza algunos de los principales desafíos ambientales y sociales a los que nos enfrentamos en la actualidad. Entre los principales retos ambientales se analizan la gestión del agua, la gestión de la biodiversidad, la gestión de los bosques y el uso del suelo y la gestión de las zonas costeras y marinas. Entre los retos sociales se presentan el conocimiento incompleto sobre el cambio climático y sus consecuencias, la falta de valoración del costo real de los recursos naturales, las desigualdades económicas y sociales entre los que ganan y los que pierden o la ausencia de políticas y sistemas de gobernanza adaptados a diferentes escalas.

Palabras clave: Sistemas Socio-Ecológicos,

\footnotetext{
1 Doctora en Economía Agraria y Desarrollo Rural (Universidad de Córdoba, España). Profesora titular (Universidad de Córdoba, España). Dirección postal: Edificio Gregor Mendel, planta 3era, Campus de Rabanales, Ctra N-IV, Km. 396 (Córdoba, España). Teléfono: (0034) 957218507; e-mail: mmdelgado@uco. es

2 Máster Erasmus Mundus en Desarrollo Rural (Universidad de Gante - Bélgica). Investigadora doctorante en la Universidad de Córdoba, España. Dirección postal: C/ Toledo 8 (Córdoba España). Teléfono: (0034) 957277722; e-mail: er2amalm@uco.es
}

Recursos naturales, Sostenibilidad.

\section{Clasificación JEL: Q01}

\section{Abstract}

Current development and natural resources exploitation patterns are increasing the tensions and impacts on these resources. Climate change is intensifying this situation. This paper analyses some of the main environmental and social challenges the Planet is currently facing. Among the main environmental challenges, the management of water, the management of biodiversity, the management of forest and land use, and the management of marine and coastal areas are mentioned. In relation to social challenges, the incomplete knowledge about climate change and its effects, the lack of valorisation of the real costs of natural resources, the social and economic imbalances between the winners and losers and the lack of policies and governance systems adapted to different scales are analysed.

3 Doctora (Universidad de Córdoba - España). Gestión de proyectos de la Universidad de Córdoba, España. Dirección postal: Plaza Conde de Priego № 2, 14001 (Córdoba, España). Teléfono: (0034) 957218441; e-mail: z62riric@uco.es 
Keywords: Socio-ecological systems, Natural Resources, Sustainability.

\section{JEL Clasification: Q01}

\section{INTRODUCCIÓN}

En la actualidad, el Planeta Tierra está sometido a cambios y desafíos sin precedentes. El actual modelo de desarrollo, las actividades de la sociedad moderna y el enorme crecimiento demográfico (la población mundial se ha multiplicado por más de cinco en menos de cien años), están generando una demanda cada vez mayor de recursos naturales y están incrementando los impactos directos e indirectos sobre los ecosistemas. Estos cambios no habían sido hasta ahora tan grandes, tan complejos o tan potencialmente catastróficos para la supervivencia de los ecosistemas. Otras características diferenciales son la rapidez con la que se dejan sentir sus efectos y la dificultad para predecirlos.

Frente a ello, la sociedad no cuenta con herramientas ni con modelos de adaptación capaces de ajustarse a esta velocidad. La relevancia a nivel mundial de los problemas y la necesidad de analizarlos en profundidad han hecho que en la última década se generen importantes avances en el conocimiento científico sobre los mismos.

Así, se han publicado estudios e informes como: i) los Informes de Evaluación del Milenio (Millennium Ecosystem Assessments, 2005); ii) los informes del Panel Intergubernamental sobre el Cambio Climático (IPCC,
2007); iii) el Informe sobre la Economía de los Ecosistemas y la Biodiversidad en 2010 (Sukhdev, 2010); iv) los diversos Informes de Perspectiva Mundial sobre la Diversidad Biológica (UNEP/GRID Arendal, 2007); v) los informes de Perspectiva Mundial sobre el Medio Ambiente, el quinto de los cuales fue presentado en la cumbre de Rio de 2012 (UNEP, 2012); vi) el Informe Stern presentado en 2006 (Stern, 2006); vii) el Informe sobre el Desarrollo Mundial de 2010 dedicado al Desarrollo y al Cambio Climático (World Bank, 2009) y viii) el documento Pilares Transformadores de la Investigación en Ciencias Sociales para el Cambio Global (ISSC, 2012).

Estos trabajos han aportado un mayor conocimiento sobre el cambio climático y sobre las condiciones en las que se encuentran los ecosistemas. Así, ponen de manifiesto una serie de importantes problemas a nivel mundial relacionados con la gestión de los recursos naturales. Entre los mayores desafíos ambientales que reconocen se pueden citar: la gestión del agua, la gestión de la biodiversidad, la gestión de los bosques y los cambios en el uso del suelo y la gestión de las zonas marinas y costeras.

Sin embargo, el reconocimiento de que los recursos naturales están profundamente afectados por la influencia del hombre hace que deban ser entendidos como sistemas socio-ecológicos (Anderies et al., 2004) y que en ellos deban ser analizados tanto los aspectos sociales como los físicos.

La percepción de las interacciones entre la gente y los ecosistemas, la actitud y el com- 
portamiento tanto individual como colectivo ante estos fenómenos y las reacciones y estrategias que puedan adoptar los habitantes de las zonas directamente implicadas en el uso y conservación de estos recursos, son temas menos analizados que los relativos a los aspectos físicos y ecológicos, aunque no por ello se enfrentan a retos menos importantes.

Ante este escenario, el objetivo del presente artículo es plantear algunos de los desafíos ambientales y sociales en el actual escenario de cambio climático, que muy previsiblemente afectarán tanto a la disponibilidad de recursos, como a sus posibles usos.

\section{PRINCIPALES DESAFIOS MEDIOAMBIENTALES}

Los recursos naturales son soportes clave para la vida en nuestro planeta. No obstante, en la actualidad se está haciendo un uso insostenible de ellos que pone en peligro su supervivencia y su disponibilidad para las generaciones futuras (CBD, 2010).

Estos recursos se concentran principalmente en las áreas rurales, las mismas que por ello se encuentren especialmente afectadas por su uso y evolución. Entre los principales desafíos medioambientales actuales en estos territorios se pueden mencionar: la pérdida de biodiversidad, las dificultades en la gestión del agua, la deforestación y la presión para cambiar el uso del suelo, y la sobreexplotación de la pesca y de otros recursos naturales.

\subsection{La pérdida de biodiversidad}

La actual biodiversidad es el resultado de billones de años de evolución de procesos naturales y de una creciente influencia de procesos antropogénicos. Los beneficios derivados de la misma van mucho más allá de la provisión de materias primas. La seguridad alimentaria, el acceso a las fuentes de energía o la vulnerabilidad ante catástrofes naturales, como los incendios o las inundaciones dependen del manejo que se haga de esta biodiversidad. El Millennium Ecosystem Assessment (2005) y The Economics of Ecosystems and Biodiversity (TEEB) (Sukhdev, 2010), demuestran que los beneficios de su conservación y uso sostenible son mucho mayores que lo que supondría su restauración, en los casos en que fuera posible.

No obstante, diversos factores, entre los cuáles el comportamiento humano juega un papel clave, están llevando al sistema Tierra a un punto crítico. La humanidad y la naturaleza están inevitablemente unidas en lo que Raudsepp-Hearne et al. (2010) Ilaman la "Paradoja Ambientalista" (Environmentalist's Paradox) según la cual el bienestar humano se incrementa a la vez que la situación del medio ambiente se degrada.

El mundo está experimentando unas tasas de extinción de especies sin precedentes. Cada día se pierde biodiversidad a una tasa que supera en mil veces la tasa natural (Diversitas, 2010). Entre las razones para ello están la extinción de especies individuales que forman parte de la cadena alimentaria de otras, la 
destrucción de hábitats, la ampliación de la frontera agrícola con tierras sin este tipo de vocación, la contaminación o la diseminación de especies invasivas. Esta escalada en la velocidad de extinción plantea dudas sobre las posibilidades de la naturaleza para soportar estas presiones. Como señalan Rockström et al (2009) se está llegando a los límites en lo que se refiere a la pérdida de biodiversidad según el concepto de planetary boundaries introducido por estos autores.

Pero además, esta situación tiene un elevado precio económico. El costo de la pérdida de biodiversidad y de servicios ecosistémicos en el planeta ha sido estimado en 50 billones de euros anuales en el periodo 2000-2010 y de seguir con los modelos y pautas de funcionamiento actuales, se prevé que esta cifra alcance los 275 billones de euros (7\% del PIB mundial) en 2050 (Sukhdev, 2010).

Frenar estos procesos de destrucción de biodiversidad fue uno de los objetivos de declarar el año 2010 como Año Internacional de la Biodiversidad. No obstante, la Conferencia de Nagoya celebrada en esta ciudad japonesa para clausurar el acontecimiento confirmó que ni los compromisos conseguidos en la misma ni los progresos de los últimos años permiten augurar un cambio en las tendencias descritas. Como fue puesto de manifiesto, el objetivo acordado por los gobernantes mundiales en 2002 de "conseguir para el año 2010 una reducción significativa de la actual pérdida de biodiversidad a escala global, regional y nacional como una contribución a la lucha contra la pobreza y un beneficio para la vida en el planeta Tierra" no ha sido conse- guido, ni parece que vaya a serlo en el futuro próximo.

Estos procesos de extinción no sólo son irreversibles, sino que además plantean serias amenazas a la salud y el bienestar humanos. La pérdida de biodiversidad afecta a la producción de alimentos, a los recursos forestales, a la disponibilidad de energía y medicinas, a las actividades turísticas y a las oportunidades de disfrute de la naturaleza.

\subsection{La gestión del agua}

Los recursos hídricos son esenciales para la vida humana, así como para el desarrollo económico y el equilibrio en la Tierra, cuya denominación de "planeta azul", se debe precisamente a la cantidad de agua que lo compone. Sin embargo, sólo una pequeña proporción de agua (en torno a un 2\%) es agua dulce, apta y accesible para el consumo humano. Además, un problema adicional es la disponibilidad de la misma. Además, mientras algunas zonas del planeta reciben más agua de la que necesitan sus habitantes, como ocurre en muchas áreas de Latinoamérica, otras zonas de África viven en un estado casi permanente de sequía.

La importancia de este recurso llevó a que entre los Objetivos de Desarrollo del Milenio se incluyera la necesidad de eliminar la explotación insostenible del agua y el desarrollo de estrategias de gestión sostenible que permitan un acceso adecuado y justo para todos los habitantes del planeta. Sin embargo, el $4^{\circ}$ Informe Mundial sobre Desarrollo del Agua, que lleva por título Gestionar el agua en 
un contexto de incertidumbre y riesgo (4th World Water Development Report. Managing Water under Uncertainty and Risk) presentado en el $6^{\circ}$ Foro Mundial del Agua en 2012 en Montpellier puso de manifiesto tendencias alarmantes en relación con la disponibilidad y acceso al agua y con los impactos que ello tiene sobre los servicios ecosistémicos y el desarrollo humano.

El consumo humano y la producción agraria, industrial y energética harán que la demanda de agua alcance volúmenes históricos. Para 2050 la demanda de alimentos se incrementará en un $70 \%$, lo que inducirá a un incremento del $19 \%$ en el uso de agua por parte del sector agrario. Este sector es de lejos el mayor consumidor de agua, representando un $70 \%$ del consumo global. Por otro lado, para 2035, el agua necesaria en otros procesos productivos se incrementará en un $50 \%$ y será clave para el crecimiento de la población y el desarrollo de actividades económicas.

Tampoco se puede olvidar que en la actualidad 1.000 millones de personas no tienen acceso a agua potable o que actualmente hay más gente en las ciudades sin acceso a agua corriente de la que había en los noventa. Se espera que en 2050, la población urbana alcance los 6.300 millones de habitantes frente a los 3.400 millones de 2009 (UNESCO, 2012).

Estas crecientes demandas de agua derivadas del incremento de población, del desarrollo de actividades económicas (industria, minería, producción eléctrica, etc.) y por la mejora de los estándares de vida en los países emergentes, crearán tensiones aún mayores por el uso y control de este recurso.

A ello se une la variabilidad, vulnerabilidad e incertidumbre del agua disponible en el tiempo y en el espacio. El agua dulce se distribuye erráticamente de un año para otro y de un lugar a otro por lo que los planes de manejo de agua tienen que incorporar esta variabilidad. Pero además de la cantidad de agua importa su calidad, la misma que, si es baja, conlleva elevados costos económicos, incluyendo la degradación de los servicios ecosistémicos, problemas de salud humana, impactos en actividades económicas e incrementos en los costos de tratamiento y depuración.

El agua, además, es un componente integral del cambio climático y el principal medio a través del cual se hace sentir sus impactos. Las perspectivas de futuro no son halagüeñas ya que se espera que el cambio climático intensifique la crisis del agua. Como establece el informe del International Panel of Climate Change (IPCC,2007) se prevé que el cambio climático exacerbe la escasez de agua, incremente la frecuencia e intensidad de eventos extremos como la sequía o las inundaciones y aumente la salinización de los acuíferos por el incremento del nivel del mar.

Los efectos se dejarán sentir a diferentes niveles. Desde un punto de visto medioambiental, los ecosistemas de agua dulce son los que están sometidos a mayores amenazas de desaparición de especies debidas al calentamiento del agua, a la alteración de flujos o a la desaparición de hábitats acuáticos (Millenium Ecosystems Assessment, 2005). 
Desde un punto de vista económico, se incrementará su nivel de contaminación y el uso y explotación del agua serán más difíciles o más costosos. Sectores tan importantes como la agricultura, la industria, el transporte, el suministro de energía (sobre todo hidroeléctrica), la pesca y el sector forestal, se verán enormemente afectados por estos cambios.

Finalmente, los efectos del cambio climático sobre los recursos hídricos se dejarán sentir también en aspectos como la salud humana o las facilidades de los ecosistemas para proveer de servicios esenciales para la vida. No obstante, lo que realmente ocurrirá representa una de las mayores incertidumbres a las que se enfrenta la humanidad en la actualidad. Las predicciones globales tienen bastante probabilidad de cumplirse, pero los efectos a nivel local son mucho más impredecibles.

\subsection{El manejo de los bosques y el uso del suelo}

Los bosques desempeñan un papel crítico en el equilibrio global del clima y en los sistemas de producción y consumo sostenibles. Realizan funciones como la captación de $\mathrm{CO}_{2}$, ser hábitat de la mayor parte de la biodiversidad terrestre del planeta (incluidas más de la mitad de las especies mundiales de animales, pájaros e insectos), y del desarrollo de importantes actividades económicas (recursos madereros, frutos, productos medicinales, alimentos, fibras...); así como la mitigación de las emisiones de $\mathrm{CO}_{2}$ a la atmósfera y la provisión de servicios ambientales. Además, la superficie forestada es clave para mante- ner la fertilidad de las tierras agrícolas, para proteger los recursos hídricos y para reducir el riesgo de desastres naturales como inundaciones o deslizamientos de tierras (FAO, 2006; FAO, 2012). Todas estas razones están en el origen de que 2011 fuese declarado como el Año Internacional de los Bosques, por parte de Naciones Unidas.

La historia humana, es una historia de los bosques y su uso. No obstante, pocas sociedades han tenido éxito en su manejo sostenible. La historia de la civilización es también una historia de deforestación. A pesar de su importancia estratégica, los bosques están siendo sometidos a unas enormes presiones que cuestionan su supervivencia. De acuerdo con FAO (2012), los bosques cubren en la actualidad el $31 \%$ de la superficie terrestre. De ellos, sólo un tercio son bosques primarios y más del $60 \%$ están seriamente degradados o son usados de manera insostenible. Más del 50\% de los bosques de distinto tipo, de las tierras agrarias y de los humedales que rodean a las zonas urbanas o periurbanas, se han perdido por la conversión del suelo a otros usos.

Además, en los últimos sesenta años la superficie forestal se ha reducido en un $60 \%$ y las dos terceras partes de lo que queda están fragmentadas, lo que hace más probable que desaparezcan también (MEA, 2005; The Economist, 25 Sep 2010). Esta situación se ha acelerado en la última década, en la que se han perdido más de 130 millones de hectáreas de bosque, 40 millones de ellas de bosques primarios (FAO, 2010a). La destrucción de bosques en algunos países de la zona Asia-Pacífico está ocasionando pérdidas de biodiversi- 
dad de entre el 2 y el $5 \%$ por década, ocasionando unos daños totalmente irreversibles a la sostenibilidad de estos ecosistemas y al bienestar humano.

A la importancia medioambiental mencionada se une su importancia socioeconómica. Normalmente, el valor económico de los bosques se suele contabilizar por el valor de la madera que producen y cuya explotación y venta está originando la desaparición de muchos bosques vitales para el planeta. Sin embargo, no se puede olvidar que en torno a 60 millones de personas (principalmente en tribus indígenas) viven directamente en y de los bosques, otros 350 millones viven en las zonas adyacentes y sus medios de vida e ingresos están ligados en una alta proporción a los mismos y más de 1.000 millones de personas usan medicinas extraídas de las especies que viven en los bosques o dependen de los recursos que albergan para necesidades vitales (FAO, 2009).

La deforestación y el cambio en el uso del suelo (principalmente de forestal a agrícola) representan amenazas muy importantes a las estrategias de mitigación de los efectos del cambio climático a nivel global y a la supervivencia de las comunidades rurales que viven en ellas.

Se estima además que el costo para la economía global de la pérdida y degradación de los bosques ha sido estimado entre 2 y 4,5 trillones de dólares anuales (Sukhdev, 2010). Desafortunadamente, estos costos no son recogidos en los indicadores tradicionales de progreso económico, como el Producto Inter- no Bruto (World Bank, 2011).

\subsection{La gestión de los océanos y las zonas costeras}

Los océanos cubren el 70\% de nuestro planeta, contienen el $97 \%$ del agua de la Tierra y su volumen representa el $99 \%$ del espacio vivo del planeta. Albergan una enorme riqueza de biodiversidad y recursos genéticos marinos. Se han identificado unas 200.000 especies, pero puede haber millones aún desconocidas. Proveen de un elevado número de servicios ecosistémicos. Son la mayor fuente de proteínas a nivel mundial y para más 2.600 millones de personas son su principal fuente de alimentación (UNEP, 2012). Además juegan un papel clave en la regulación del clima global, ya que entre otras funciones tienen una capacidad de almacenamiento de $\mathrm{CO}_{2}$ quince veces superior a la que tienen la biosfera y el suelo terrestres, absorbiendo más del $30 \%$ del $\mathrm{CO}_{2}$ producido por las actividades humanas $\mathrm{y}$ generando un importante efecto tampón de los impactos del calentamiento global.

Desde un punto de vista económico, unos 3.000 millones de personas dependen de la biodiversidad marina y costera para su sustento. El valor de mercado de los recursos e industrias costeras es estimado en 3 trillones de dólares al año, lo que representa el $5 \%$ del PIB mundial (UNEP, 2012).

A pesar de estas funciones, en torno al $40 \%$ de los océanos mundiales están altamente afectados por la actividad humana. Entre las 
principales amenazas a las que se enfrentan se pueden destacar la sobrepesca, las técnicas de pesca destructivas, la contaminación, la destrucción de hábitats de diferentes especies, la presencia de especias invasivas, el incremento en el tráfico marino (que está creciendo a una media anual del 9-10\%) o los riesgos de vertidos de distinto tipo, pero con especial incidencia los petrolíferos (UNEP, 2010).

Las estimaciones apuntan a que el cambio climático acentuará estas amenazas y empeorará esta situación. Los océanos serán los primeros en sentir los efectos del calentamiento global. Los cambios de temperatura y salinidad tendrán efectos importantes en las especies marinas. Las modificaciones en la disponibilidad de fitoplancton originarán efectos en cascada a lo largo de la cadena trófica que serán más patentes en las zonas más dependientes de la pesca. Pero sobre todo, ocasionarán cambios muy importantes en la circulación de las masas de agua que afectarán a las condiciones climáticas tanto a escala local, en zonas específicas, como a escala global.

UNEP (2010) advierte sobre el riesgo de deterioro significativo de la biodiversidad marina en los próximos veinte años. El ritmo de incremento de la pesca es insostenible. Los subsidios a la pesca están contribuyendo a la rápida destrucción de muchas especies marinas y están frenando los esfuerzos para salvar y restaurar pesquerías, causando pérdidas globales de unos 50 billones de dólares al año (UNEP, 2012). La proporción global de especies que se pescan en exceso no ha dejado de incrementarse en los últimos 25 años, aunque el fenómeno se ha ralentizado. Las capturas se incrementaron de 19 millones de toneladas en 1950 a unos 80 millones de toneladas a mitad de los ochenta. Estas cifras oscilan en la actualidad en torno a los 90 millones de toneladas (FAO, 2010b). Esta estabilización no se ha debido a una conciencia sobre la insostenibilidad de las prácticas de pesca sino a que el incremento de la tasa anual de capturas marinas se situó en el entorno de cero en los noventa, indicando que, en promedio, los océanos habían alcanzado su máxima capacidad de producción bajo los sistemas de extracción actuales.

Europa es el continente más afectado por estos problemas. La falta de una adecuada gestión de las pesquerías (elevado número de barcos, altas cuotas y largas temporadas de pesca o escaso control sobre la pesca de alevines) hace que más del $72 \%$ de los bancos de pesca existentes estén sobreexplotados, frente a un $25 \%$ del promedio global (Froese y Proels, 2010).

No obstante, donde los efectos del cambio climático van a ser más intensos es en las zonas costeras que están sometidas a muchos más impactos antropogénicos que las zonas oceánicas.

Las zonas costeras constituyen uno de los hábitats más dinámicos del planeta y están sujetas a continuos impactos de diferente tipo (Perillo y Piccolo, 2011). Desde el punto de vista humano, se estima que la densidad de población en las zonas costeras a nivel mundial se duplicará para 2050, con un incremento de en torno al 50\% entre 2010 y 2050 (Syvistki et al., 2005). Además, 24 de las 39 ciudades con 
más de 5 millones de habitantes del planeta están situadas a menos de $100 \mathrm{~km}$ de la costa, siendo 12 de ellas megaciudades con más 10 millones de habitantes (Nicholls et al., 2007). Muchas de estas ciudades pueden tener graves problemas si se cumplen las previsiones existentes sobre el incremento del nivel del mar.

Los hábitats de estas zonas están sometidos a unos enormes impactos derivados de la expansión urbana y la industrialización, y de la contaminación que llevan asociadas. Ello está originando intensas modificaciones, muchas permanentes, ya que sobrepasan el umbral de retorno a la situación original, afectando a las dinámicas costeras, a su producción primaria y a la cadena trófica asociada (MacCraken et al., 2009). Estos cambios se dejarán sentir especialmente sobre las comunidades que viven de los recursos marinos y de la pesca artesanal.

\section{DESAFIOS SOCIALES}

Los ecosistemas naturales y las sociedades humanas no pueden ser considerados de forma independiente, sino unidos en sistemas socio-ecológicos (Anderies et al., 2004). Una adecuada respuesta y una gestión sostenible de los recursos naturales y de los desafíos mencionados en el anterior epígrafe requiere el reconocimiento de que son parte de sistemas socio-ecológicos complejos y muy dinámicos, que evolucionan a menudo de forma inesperada y no lineal, en función de las intervenciones naturales y humanas que reciben (Nelson et al., 2007). El medio ambiente y los recursos naturales condicionan y son simul- táneamente condicionados por las acciones ejercidas por la población.

De ahí que cada vez sea más importante analizar los aspectos sociales que afectan a la gestión de estos recursos en un escenario de cambio climático y que también constituyen importantes desafíos. Entre los más relevantes se pueden citar el conocimiento incompleto sobre el cambio climático y sus consecuencias, la falta de valoración de los costos reales, las desigualdades económicas y sociales entre los que ganan y los que pierden o la ausencia de políticas y sistemas de gobernanza adaptados a diferentes escalas.

\subsection{Un conocimiento incompleto}

El conocimiento científico actual sobre el cambio climático está sometido a informaciones contradictorias, pero sobre todo a incertidumbres. El incompleto conocimiento de la complejidad y variabilidad de los sistemas socio-ecológicos, la limitada disponibilidad de información (debido a que el acceso a la misma es muy caro, porque se necesita mucho tiempo para obtenerla o por la falta de registros y referencias temporales sobre lo que ha ocurrido en nuestro planeta) y las incertidumbres en cuanto a los modelos y las respuestas de los ecosistemas a las intervenciones humanas, son algunas de las razones que lo explican.

También es importante entender la naturaleza transitoria de muchos de los conocimientos que se están generando, ya que tanto los 
avances en el conocimiento, como la velocidad y naturaleza de los cambios así lo exigen.

Los enfoques simplistas y monodimensionales basados en las metodologías de trabajo y el conocimiento de una única disciplina no son capaces de abarcar la complejidad de estos sistemas. Tradicionalmente, las ciencias naturales, las sociales y dentro de estas la economía, han evolucionado de forma diferenciada, sin buscar puntos de encuentro ni reconocer o combinar los conocimientos derivados de cada una de ellas. Ello hace que a pesar de los esfuerzos por abordar estas complejas problemáticas de manera multidisciplinaria y de los logros conseguidos, aún quede mucho por hacer. Las dificultades para que los científicos de diferentes disciplinas aborden conjuntamente los desafíos ambientales que plantea el cambio climático son muy altas.

La situación es aún más complicada cuando se analiza la sociedad civil, la misma que se enfrenta a informaciones contradictorias, incompletas o poco transparentes sobre la complejidad y las causas del cambio climático. Ello introduce dificultades para entender y prever los resultados y las posibles respuestas a las acciones que se están ejerciendo sobre los recursos naturales y los ecosistemas. Los ciudadanos muy a menudo tampoco disponen de las capacidades necesarias para adaptarse a los nuevos escenarios. De hecho, la ausencia de estas capacidades se configura como una de las barreras más importante en las estrategias de mitigación y adaptación al cambio climático.
Otro desafío importante es unir el conocimiento científico y el conocimiento local existentes en torno a los desafíos ambientales y el cambio climático. La relevancia y validez del conocimiento local ha sido demostrada por diferentes estudios (Orlove et al., 2000; Riedlinger and Berkes, 2001; Berman and Kofinas, 2004). Sin embargo, como señalan Cruikshank (2001) y Berkes (2002) este conocimiento no se ha integrado o se ha hecho de forma descontextualizada en los modelos y predicciones científicos. Raymond et al. (2010) ponen de manifiesto las dificultades de integrar distintos tipos de conocimiento y la necesidad de pasar de desarrollar productos para la integración de conocimientos, a procesos de integración de conocimientos.

Es necesario desarrollar marcos de entendimiento que permitan tanto reconocer el valor del conocimiento local por parte de los científicos, como hacer asequible el conocimiento científico para que la sociedad pueda contextualizar lo que está sucediendo y actuar en consecuencia. Para avanzar en estos aspectos es necesario poner en marcha estrategias de acción comunicativa y procesos de aprendizaje social que involucren a científicos, expertos, políticos y sociedad civil (Rist et al., 2007). Así, se podría avanzar en la creación de un conocimiento y entendimiento compartidos y en la transformación de las percepciones y actuaciones.

Finalmente, el conocimiento está mucho más desarrollado a escala global que a escala local. Los estudios, informes y análisis mencionados se refieren al planeta en su conjunto o a grandes bloques regionales. No obstante, 
el conocimiento de los fenómenos y sus repercusiones a nivel local es mucho más incompleto. Las diferencias y la complejidad de cada ecosistema dificultan su comprensión y su conocimiento. Las particularidades que afectan a cada uno de ellos tanto a nivel ecológico como a nivel humano también crean dificultades para extender los conocimientos o para aplicarlos a contextos o escalas diferentes.

\subsection{La falta de valoración del costo real de los recursos naturales}

El poder de los mercados y su hegemonía en el modelo económico dominante ensalzan al valor económico frente a cualesquiera otros valores. Los recursos naturales y los servicios ecosistémicos son claves para el funcionamiento y la vida en el Planeta, como ya se ha comentado. Sin embargo, en la mayoría de las ocasiones el hecho de que no exista una valoración ni un precio de mercado de estos bienes y servicios hacen que no se reconozca su valor.

La mayoría de estos servicios, como la disponibilidad de agua, la captación de $\mathrm{CO}_{2}$ realizada por los bosques, o el poder respirar aire limpio no son considerados como servicios de alto valor. Frente a esta concepción, el TEEB ha calculado el costo del daño realizado por la actividad humana a la naturaleza en 2008 en un rango entre 2 y 4,5 trillones de dólares (Sukhdev, 2010).

Estos bienes y servicios entran dentro de la consideración de bienes públicos, es decir, no existe rivalidad (el bien no desaparece en el proceso de consumo) ni posibilidades de exclusión (no es posible excluir del disfrute del bien a otros usuarios) y como tales están sometidos a la denominada 'tragedia de los comunes' (tragedy of commons) (Hardin, 1968). Esta situación hace que no haya unos derechos de propiedad claramente establecidos sobre los mismos y tampoco sea fácil identificar y penalizar a los individuos o empresas que causan daños. Los bienes públicos de acceso abierto y difícil control (como los que se encuentran en alta mar o los bosques públicos) están entre los ecosistemas más vulnerables.

Por otro lado, también es posible identificar diferentes estrategias para conservar estos recursos, especialmente por parte de comunidades locales de países en desarrollo. Las formas de organización tradicionales y el respeto por la naturaleza, les llevan a realizar una gestión ambientalmente sostenible de los recursos naturales. De esta manera contribuyen a la generación de bienes y servicios ambientales, pero no reciben ningún reconocimiento económico ni valor añadido por su gestión. Al contrario, suelen ser comunidades bastante pobres y con limitados medios de vida. No existen mecanismos efectivos para hacer que estas comunidades obtengan beneficios por proveer de bienes y servicios ambientales de alto valor para la humanidad. Las estrategias de pagos por servicios ambientales están aún poco desarrolladas y no suponen ingresos significativos para los 'productores' de estos servicios. 
Otro aspecto importante a analizar en cuanto a la valoración de los recursos naturales es la falta de información y transparencia sobre los costos reales de muchas de las actividades que se realizan diariamente. El actual estilo de vida y el rápido crecimiento económico buscado por la mayoría de los países están llevando a niveles insostenibles de degradación de los ecosistemas naturales, de la que en muchas ocasiones los ciudadanos no son conscientes. Las crecientes necesidades de consumo, el incremento de los niveles de riqueza y bienestar personales y el desarrollo tecnológico permiten a los ciudadanos de los países desarrollados y emergentes demandar y consumir un rango cada vez mayor de bienes y servicios. Sin embargo, estos beneficios se producen con unos costos ambientales que no son normalmente conocidos ni transparentes para los consumidores.

La huella de agua o de carbono de aspectos tan cotidianos como el consumo de carne, de productos de fuera de temporada o la demanda de productos procedentes de lugares lejanos no es apenas conocida por los consumidores ordinarios, ya que por un lado, no forma parte de las preocupaciones diarias, y por otro, ni los costos sociales ni los ambientales de estos bienes y servicios suelen ser incluidos en su precio.

\subsection{Las desigualdades económicas y sociales entre los que ganan y los que pierden}

Otro de los desafíos sociales importantes es el que tiene que ver con la desigual distribución entre los que ganan y los que pierden con el cambio climático y la destrucción de los recursos naturales. La mayoría de los recursos naturales de alto valor se encuentran situados en países en desarrollo y en las áreas rurales de estos países, en las que los niveles de pobreza son muy altos (IFAD, 2010).

La gente que más pierde con la degradación o desaparición de dichos recursos es aquella cuyo sustento depende directamente de los mismos o vive en zonas aledañas. Como afirmó Sukhdev, líder del TEEB, "la biodiversidad tiene valor para todos, pero es una absoluta necesidad para los pobres" (Informe de prensa de la Conferencia de Nagoya, 2010).

En la Conferencia Inaugural de Nagoya 2010, Ban Ki-moon, Secretario General de Naciones Unidas también se pronunció en este sentido: "la pérdida de biodiversidad está llevando a los sistemas ecológicos a un punto de no retorno, más allá del cual no será posible que desarroIlen sus funciones vitales... Las comunidades de todo el mundo sufrirán sus consecuencias negativas, pero los pobres y los más vulnerables serán los que las sufran en mayor medida".

Los medios de vida y el trabajo de cientos de millones de personas dependen de la existencia y disponibilidad de estos recursos. Además, los impactos físicos más duros del cambio climático (sequías, inundaciones, tormentas tropicales, olas de calor...) están teniendo una especial incidencia en los países de menores ingresos y más vulnerables (World Bank, 2009). 
Una gestión inadecuada e insostenible de los recursos naturales en estos países no sólo creará problemas ambientales a nivel local y global, sino que tendrá importantes implicaciones en otros temas como la pobreza, las migraciones, la falta de oportunidades de desarrollo, etc. que no pueden ser despreciados.

La acción colectiva para identificar e implementar una gestión sostenible de los recursos naturales es más necesaria dónde la gente tiene incentivos más débiles para tener en cuenta el impacto de sus acciones. Esta acción colectiva también es más necesaria donde los servicios en riesgo tienen un alto valor social.

Sin embargo, los ciudadanos de los países más desarrollados ven estos problemas muy lejanos y poco relacionados con sus problemas cotidianos. La desconexión de la naturaleza que impera en países desarrollados y en el mundo urbano hace que se valoren bienes que llevan un elevado consumo energético y de otro tipo de recursos y que no se tenga en cuenta la destrucción de recursos, culturas o medios de vida que ello puede conllevar.

\subsection{La falta de políticas y sistemas de gobernanza adaptados a distintas escalas}

Las discusiones globales, las cumbres y las iniciativas internacionales pueden despertar gran atención a nivel global. Sin embargo, no se puede olvidar que aunque los problemas y los desafíos son globales, sus efectos se dejan sentir a nivel local. Es necesario contar con políticas y estrategias adaptadas a las situaciones locales y al manejo sostenible de los recursos a nivel local.

En la mayoría de los casos, el capital natural y los servicios ecosistémicos son específicos de un área concreta y es a ese nivel al que deben ser gestionados y preservados. Como señala The Economist (21 de octubre de 2010), "las discusiones globales están muy bien, pero la mayoría de las buenas prácticas de conservación se hacen a nivel local".

Hasta ahora, las diferentes cumbres y conferencias internacionales están consiguiendo atraer la atención del público y de los gobernantes sobre el cambio climático y la degradación de los recursos naturales, pero no llevan aparejadas una toma de decisiones efectiva. Al no existir un sistema de gobernanza global ni de cesión de soberanía mundial no se puede obligar a cumplir los compromisos relativos al cambio climático o a la degradación de recursos naturales, ni forzar a los países que más están incidiendo en la degradación de los recursos o en el cambio climático a cambiar de estrategias.

Ante esta situación, se plantea la necesidad de pasar de las declaraciones de buenas intenciones al establecimiento de metas y compromisos reales, que además incorporen no sólo a los gobiernos y al sector público, sino también al sector privado y a la sociedad civil, y a partir de ahí, instaurar sistemas de gobernanza efectivos que permitan su cumplimiento.

Algunas metas, sólo podrán ser conseguidas 
por la suma de los esfuerzos individuales de los diferentes países. Otras necesitarán la colaboración de los países a través de acuerdos y con la implicación de agencias internacionales (Perrings et al., 2010). Sin embargo, dichas metas y compromisos no podrán ser conseguidos sin la implicación y participación de las comunidades en las que se sitúan los recursos.

Una adecuada y eficiente participación de la población es imprescindible para entender los aspectos involucrados en la relación entre la gente y el medio ambiente (Pretty and Smith, 2004), como demuestra la existencia de modelos de gobernanza exitosos y sostenibles cuando las comunidades locales tienen acceso y control sobre los recursos naturales (Dietz et al., 2003; Ostrom, 2005). No obstante, los problemas más importantes se plantean, cuando estas reglas no son respetadas ni reconocidas ni por otros actores, ni por niveles administrativos superiores.

La gestión de los recursos naturales, es ante todo una cuestión de gobernanza. Es necesario disponer de reglas y sistemas de manejo tanto a nivel local, como de interacción de ese nivel local con niveles superiores.

\section{REFLEXIONES FINALES}

Las llamadas de atención sobre la situación actual de los recursos naturales, la degradación a la que están siendo sometidos y sus consecuencias en el mediano y largo plazo tienen ya una larga trayectoria. Sin embargo, hasta ahora ni la opinión pública ni la mayoría de los gobiernos han tomado medidas efectivas para paliarlas. Identificar opciones para el manejo sostenible de estos recursos, en un escenario de cambio climático es una necesidad cada vez más urgente.

Los vínculos entre la situación del medioambiente, la economía y el bienestar humano nunca han sido tan obvios como lo son en estos momentos. A pesar de ello el papel de los servicios ecosistémicos soportando la vida en la Tierra y proveyendo de medios de vida y oportunidades a sus millones de habitantes no es reconocido. Se hace necesario entender y reconocer el alto valor de los recursos existentes en los hábitats naturales y su carácter imprescindible para la vida y el desarrollo en el Planeta.

Las soluciones que se propongan no pueden abordar los temas ambientales y los temas sociales como esferas desconectadas. El mundo actual está formado por complejos sistemas socioecológicos en los que existe una densa red de interacciones e interdependencias entre sus componentes humanos y naturales. Las decisiones de manejos, tanto sostenibles como insostenibles, son tomadas por las personas y las mismas se dejan sentir sobre los recursos naturales.

Las relaciones entre los ámbitos ambiental y humano y las interdependencias e influencias que se generan entre ambos necesitan ser analizadas en más profundidad y nuevos conocimientos son necesarios. Los análisis y las propuestas han de hacerse desde enfoques multidisciplinares que permitan avanzar en el conocimiento real de lo que está ocurriendo y 
también establecer puentes de conexión (en ambos sentidos) entre el conocimiento científico y el conocimiento local.

Un mayor esfuerzo investigador es necesario para identificar y eliminar las barreras entre el conocimiento desarrollado por diferentes disciplinas y entre el conocimiento científico y local. Es importante profundizar en el conocimiento de los efectos e impactos a nivel local e intensificar los esfuerzos en difundir buenas prácticas y lecciones de interés.

Los problemas han de ser abordados a la escala más adecuada y a partir de ahí establecer objetivos y metas para cada escala. Las soluciones globales han de tener en cuenta la realidad y las prácticas existentes a nivel local, nacional o de bloques regionales y actuar en consecuencia, y el manejo a nivel local no puede obviar las políticas o los acuerdos nacionales o globales.

Los nuevos modelos de gobernanza a nivel local y global que se propongan deben minimizar los factores de estrés antropogénicos (contaminación, deforestación, sobrepesca, erosión y alteraciones de la hidrología, pérdida de suelos y de su fertilidad, etc.) e implementar estrategias de adaptación ambientales, sociales y económicas adecuadas. Las propuestas de políticas y la toma de decisiones deben entender los sistemas de gobernanza existentes en las distintas escalas, para asegurar que unas no entran en contradicción con otras.

Las actuales prácticas de manejo y gestión han de ser ajustadas en términos temporales, situacionales, de escala de trabajo y de coordinación de acciones para poder apoyar la resiliencia de los ecosistemas en el contexto de rápido cambio climático. Además, es imprescindible incluir las percepciones sociales y las actitudes en el análisis del cambio climático y en la forma de abordar estrategias de mitigación y de adaptación.

\section{AGRADECIMIENTOS}

Esta investigación ha sido financiada por el 70 Programa Marco de la Unión Europea en el marco del proyecto "Community based management of environmental challenges in Latin America" (FP7-ENV2011-282845 COMET-LA). 


\section{BIBLIOGRAFÍA}

Anderies, J.M., Janssen M.A. \& E. Ostrom (2004). A framework to analyze the robustness of socioecological systems from an institutional perspective. Ecology and Society 9(1), 13-34.

Berman, M. \& Kofinas, G. (2004). Hunting for models: grounded and rational choice approaches to analyzing climate effects on subsistence hunting in an Arctic community. Ecological Economics 49, 31-46.

CBD (2010). Convention on Biological Diversity. http://www.cbd.int/. Consultada el 30 Oct 2010.

Cruikshank, J. (2001). Glaciers and climate change: perspectives from oral tradition. Arctic 54, 377-393

Dietz T., Ostrom E. \& Stern P. C (2003). The Struggle to Govern the Commons. Science 302 (5652), 1907 - 1912.

Diversitas (2010) DIVERSITAS at CBD-COP10. http://www.diversitas-international.org/ uploads/File/DIVERSITAS_COP10(1).pdf. Consultada el 30 Oct 2010.

FAO (2006). Evaluación de los recursos forestales mundiales 2005: hacia la ordenación forestal sostenible (FRA 2005). Estudio FAO: Montes № 147. Roma.

FAO (2009). State of the World's Forests. FAO. Roma. 166 pp.
FAO (2010a). Global Forest Resources Assessment 2010. Main report. FAO Forestry Paper No. 163. Rome. Recuperado a partir de www. fao.org/docrep/013/i1757e/i1757e00.htm.

FAO (2010b). Estadísticas de pesca y acuicultura. Recuperado a partir de http://www.fao. org/docrep/015/ba0058t/ba0058t00.htm

FAO (2012). State of the World's Forest. Recuperado a partir de http://www.fao.org/docrep/016/i3010e/i3010e.pdf

Froese, R., \& Proels, A. (2010). Rebuilding fish stocks no later than 2015: Will Europe meet the deadline? Fish and Fisheries 11(2), 194202.

Global Biodiversity Outlook 3. http://www. cbd.int/. Consultada el 28 Dic 2011.

Global Environment Outlook for development (GEO-4). Recuperado a partir de http:// www.unep.org/geo/geo4.asp. Consultada el 28 Dic 2011.

Hardin, G. (1968). The Tragedy of the Commons. Science, 162: 1243-1248.

IFAD (2010). Rural Poverty Report 2011. New realities, new challenges: new opportunities for tomorrow's generation. IFAD, Rome.

IPCC (2007). Impacts, Adaptation and Vulnerability. Contribution of Working Group II to the Fourth Assessment Report of the Intergovernmental Panel on Climate Change, B. Metz, O. Davidson, P. Bosch, R. Dave and L. Meyer, 
Eds., Cambridge University Press, Cambridge, UK.

ISSC (2012). Transformative Cornerstones of Social Science Research for global Change. Recuperado a partir de http://www.worldsocialscience.org/?p=2871

MacCracken, M., Escobar-Briones, E., Gilbert, D., Korotaev, G., Naqvi, W., Perillo, G.M.E., Rixen, T, Stanev, E., Sundby, B., Thomas, H., Unger, D. and Urban, E.R. (2009). Vulnerability of semi-enclosed marine systems to environmental disturbances. In: Malanotte Rizzoli, P., Melillo, J, Sundby, B., Urban, E. (Eds.), Watersheds, Bays, and Bounded Seas: The Science and Management of Semi-Enclosed Marine Systems, Island Press, Washington, DC, pp. 9-29.

Millennium Ecosystem Assessment (2005). Ecosystems and Human Well-Being: Current State and Trends. Island Press, Washington, DC.

Nelson, D., Adger, W. N. and Brown, K. (2007). Adaptation to environmental change: contributions of a resilience framework. Annual Review of Environment and Resources 32, 395419.

Nicholls RJ, Hanson S, Herweijer C, Patmore N, Hallegatte S, Corfee-Morlot J, Cha`teau J, Muir-Wood R (2007). Ranking portcities with high exposure and vulnerability to climate extremes-exposure estimates. OECD environmental working paper no. 1. OECD. Paris.
Orlove B.S., Chiang J.C.H. et al (2000). Forecasting Andean rainfall and crop yield from the influence of El Nino on Pleiades visibility. $\mathrm{Na}$ ture $403,68-71$

Ostrom E. (2005). Understanding institutional diversity. Princeton University Press. Princeton.

Pauly, D., Watson, R. and Alder, J. (2005). Global trends in world fisheries: impacts on marine ecosystems and food security. Phil. Trans. R. Soc. B 360, 5-12.

Perillo, G.M.E. and Piccolo, M.C. (2011). Global variability in estuaries and coastal settings. In: Simstead, C. and Yanagi, T. (Eds.) Features/ Classification of Estuaries and Coastal Waters. Treaties on Estuarine and Coastal Science Vol. 1. Elsevier. Amsterdam.

Perrings, C., Naeem, S., Ahrestani, F., Bunker, D. E, Burkill, P., Canziani, G., Elmqvist, T., Ferrati, R., Fuhrman, J., Jaksic, F., Kawabata, Z., Kinzig, A., Mace, G. M., Milano, F., Mooney, H., Prieur-Richard, A.-H., Tschirhart, J. \& Weisser W. (2010). Ecosystem Services for 2020. Science 330, 323-324.

Pretty, J. and Smith, D. (2004). Social Capital in Biodiversity Conservation and Management. Conservation Biology, 18: 631-638.

Raudsepp-Hearne, C., Peterson G. D., Tengö M., Bennett E. M., Holland T., Benessaiah K., MacDonald G. K., and Pfeifer L. (2010). Untangling the Environmentalist's Paradox: Why Is Human Well-being Increasing as Ecosystem 
Services Degrade? BioScience 60 (8), 576-589. doi:10.1525/bio.2010.60.8.4. http://caliber. ucpress.net/doi/abs/10.1525/bio.2010.60.8.4.

Raymond, C., Fazey I., Reed M., Stringer L., Robinson G. and Evely A. (2010) Integrating local and scientific knowledge for environmental management. 91 pp. 1766-1777

Rockström, J., Steffen W., Noone K., Persson Å., Chapin F.S., Lambin E.F., Lenton T.M., Scheffer M., Folke C., and Schellnhuber H.J., others. 2009. A safe operating space for humanity. Nature 461, no. 7263: 472-475. http://www. nature.com/nature/journal/v461/n7263/pubmed/461472a.html.

Riedlinger, D. \& Berkes, F. (2001). Contributions of traditional knowledge to understanding climate change in the Canadian Arctic. Polar Record 37:315-328.

Rist, S., Chidambaranathan, M., Escobar, C., Wiesmann, U., Zimmermann, A. (2007). Moving from sustainable management to sustainable governance of natural resources: The role of social learning processes in rural India, Bolivia and Mali. Journal of Rural Studies 23, 23-37.

Stern, N. (2006). Stern Review on the Economics of Climate Change. Cambridge University Press. Cambridge.

Syvitski, J.P.M., Vörösmarty, C., Kettner, A.J. and Green P. (2005). Impact of humans on the flux of terrestrial sediment to the global coastal ocean. Science 308, 376-380.
Sukhdev (2010). The Economics of Ecosystems and Biodiversity for national and international Policy Makers. http://www.teebweb. org/. Consultada el 15 Oct 2012.

UNEP (2010) Global Synthesis. A report from the Regional Seas Conventions and Action Plans for the Marine Biodiversity Assessment and Outlook Series. UNEP Regional Seas Development.

UNEP (2012). Global Environment Outlook 5: GEO5: environment for the future we want. Nairobi. Recuperado a partir de http://www. unep.org/geo/pdfs/geo5/GEO5_report_full_ en.pdf

UNEP/GRID-Arendal. 2007. Protected areas and wilderness (http://maps.grida.no/go/graphic/ protected-areas-and-wilderness).

UNESCO (2012). 4th World Water Development Report. Managing Water under Uncertainty and Risk. Recuperado a partir de http://www. unesco.org/new/en/natural-sciences/environment/water/wwap/wwdr/wwdr4-2012/

World Bank (2009). World Development Report 2010: Development and Climate Change. World Bank, Washington.

World Bank (2011). The changing wealth of nations. Measuring sustainable development in the new millennium. Washington DC. http://publications.worldbank.org/index. php?main_page=product_info\&products_ $\mathrm{id}=23895$. 